\title{
Case report: Imaging of Corpus Callosum Agenesis
}

\author{
Kyoung-Bo Lee, PT, $\mathrm{PhD}^{1}$, Sang-Ho Lee, PT, $\mathrm{PhD}^{2}$, Seong-Hoon Lim, MD, $\mathrm{PhD}^{* 3}$ \\ ${ }^{1}$ Dept. of Physical Therapy, Catholic University, St. Vincent Hospital, Republic of Korea \\ ${ }^{2}$ Dept. of Physical Therapy, College of Health Science, Sunmoon University, Republic of Korea \\ ${ }^{*}$ Dept. of Rehabilitation medicine, Catholic University, St. Vincent Hospital, Republic of Korea
}

\begin{abstract}
Purpose Agenesis of the corpus callosum (AgCC) is one of several disorders of the structure that connects the two hemispheres of the brain. We describe two patients with AgCC and compare their magnetic resonance imaging (MRI) and diffusion tensor imaging (DTI) findings with those of healthy controls. Methods Two females with AgCC underwent brain MRI. DTI-Studio software (CMRM, Johns Hopkins Medical Institute, MD, USA) was used to reconstruct the corpus callosum (CC) fibers. Results MRI revealed periventricular plaques, complete CC agenesis of corpus callosum, and a residual anterior commissure (AC). In the DTI images of normal subjects, transcallosal fiber tracts were apparent, and formed many more connections to the frontal area than was the case in the patients. Conclusion Early diagnosis and intervention may improve the social and developmental outcomes of patients with AgCC. Symptoms and seizures must be managed. A focus on right/left co-ordination render the condition much more manageable than is otherwise the case.
\end{abstract}

Key words Agenesis of the corpus callosum, Clinical findings, MRI, Diffusion tensor imaging

Corresponding Author Seong-Hoon Lim (limseonghoon@gmail.com)

$\begin{array}{llll}\text { Purpose } & 03 & \text { Dec } 2019 \\ \text { Revised date } & 11 & \text { Feb } 2020 \\ \text { Accept date } & 11 & \text { Feb } 2020\end{array}$

\section{Introduction}

The adult corpus callosum (CC) connects the brain hemispheres and contains about 180 million axons of which $40 \%$ are myelinated. ${ }^{1)}$ The CC is completely absent in patients with agenesis of the CC (AgCC); a partial absence is termed dysgenesis. ${ }^{2)}$ AgCC is caused by disruption of one of several steps in neural development. The effects depend on the extent of brain abnormality and range from subtle through mild to severe. The latter patients may exhibit intellectual impairment, seizures, hydrocephalus, and spasticity. ${ }^{2)}$ AgCC is not uncommon; the clinical features span a spectrum and the neurological features are variable. AgCC is often associated with neurodevelopmental handicaps in children; AgCC is usually detected by neuroimaging (computed tomography, MRI, electroencephalography, and/or sonography. ${ }^{2)}$ MRI optimally reveals the cortical and subcortical architectures and

http:dx.doi.org/10.17817/2020.02.11.111503 diffusion tensor imaging (DTI) (a specific form of diffusion-weighted imaging [DWI]) evaluates restriction of perpendicular water diffusion in the brain; water motion is less constrained in other directions. DTI identifies disconnections and reveals post-stroke white-matter damage. ${ }^{3)}$ DTI images brain axonal pathways using fractional anisotropy (FA) to explore tissue microstructure. ${ }^{4)}$ Here, we describe two AgCC patients and their MRI and DTI findings to compare between T1 anatomical weighted image and tractography.

\section{Materials and Methods}

\section{Subjects \& Methods}

Two females presented with AgCC; we performed brain MRI and DTI using a 3.0-T MRI platform (MAGNETOM Verio; Siemens, Erlangen, Germany) equipped with a six-channel head coil. We acquired single-shot, spin-echo echo-planar images of axial slices covering the entire brain $[76$ 2.0-mm-thick inter- 
leaved slices (no gaps)]. The operating conditions were repetition time $(\mathrm{TR}) /$ echo time $(\mathrm{TE})=14,300 / 84$ ms; field of view $=224 \times 224 \mathrm{~mm}^{2}$; matrix $224 \times 224$; voxel size $1 \times 1 \times 2 \mathrm{~mm}^{3}$; and number of excitations $=1$. Diffusion-sensitizing gradients were applied in 64 non-collinear directions using b-values of 1,000 $\mathrm{ms} / \mathrm{mm}^{2}$. The $\mathrm{b}=0$ images were obtained prior to DWI (65 volumes in total). ${ }^{5)}$ DTI-Studio software (CMRM; Johns Hopkins Medical Institute, MD, USA) was used to reconstruct the CC fibers. Fiber tracking employed the fiber assignment continuous tracking (FACT) algorithm with an FA threshold of 0.2 and an angle threshold of $50^{\circ}{ }^{6}$; ; we drew multiple regions of interest (ROIs). ${ }^{7}{ }^{8)}$ CC tractography was performed by manually drawing ROIs on individual FA color maps; we employed the two-ROIs approach. One region was drawn on a midsagittal slice encompassing the entire CC, and the second adjacent to the cortex; these were associated with multiple projections to the orbital, frontal, parietal, occipital, and temporal lobes.
All ROIs were drawn using the anatomical landmarks and guidelines of Huang et al. ${ }^{9)}$ In addition, data of cognitive functions were collected from medical report, which are such as Korean mini-mental state examination(MMSE-K), global deterioration scale(GDS).

\section{Results}

For cases of $\mathrm{C}$ and $\mathrm{D}$, they had cognitive problems, there showed a score of 16 and 18 on the MMSE-K, respectively. It was able to have memory registration but not able to recall of memory. Also, they appeared to be low attention and visual perception problem. For GDS, it showed a score of 6 and 5, respectively.

MRI revealed periventricular plaques; parallel ventricular alignment (the 'racing car' sign) and colpocephaly were also evident. In the sagittal profile, complete CC agenesis with residual anterior commissures (ACs) were evident (Fig. 1). DTI revealed total ab-

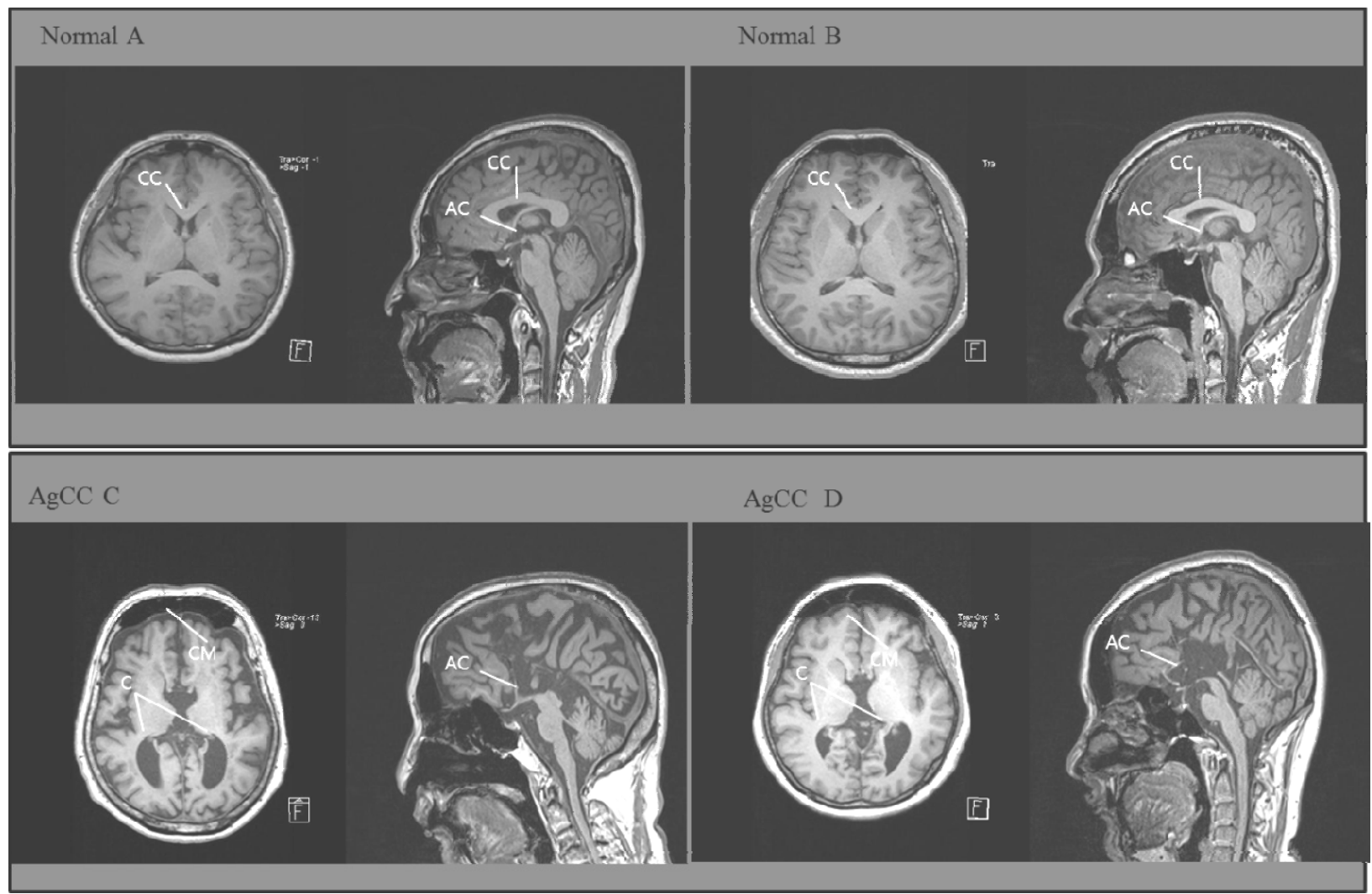

Figure 1. MRI reveals the neuroanatomical characteristics of AgCC compared to those of normal subjects. Structural T1-weighted MRI (axial and sagittal images) of a normal male and female (top) and AgCC females (bottom). AC, anterior commissure; C, colpocephaly; CC, corpus callosum; CM, cortical malformation. 
sence of the CCs (Fig. 2). In the DTI image of a normal subject, the transcallosal fiber tracts are color-coded by reference to their projections (orbital lobe, blue; frontal lobe, green; parietal lobe, orange; occipital lobe, yellow; and temporal lobe, cyan. A large amount of the $\mathrm{CC}$ was connected to the frontal area (Fig. 3).

\section{Discussion}

The CC is the largest structure connecting the brain hemispheres. ${ }^{10)}$ It remains unclear whether the connections are primarily excitatory or inhibitory. If they are excitatory, ${ }^{11)}$ brain asymmetries will be in play; the hemispheres will function independently. ${ }^{12)}$ AgCC is associated with severe deficits in cognition and social
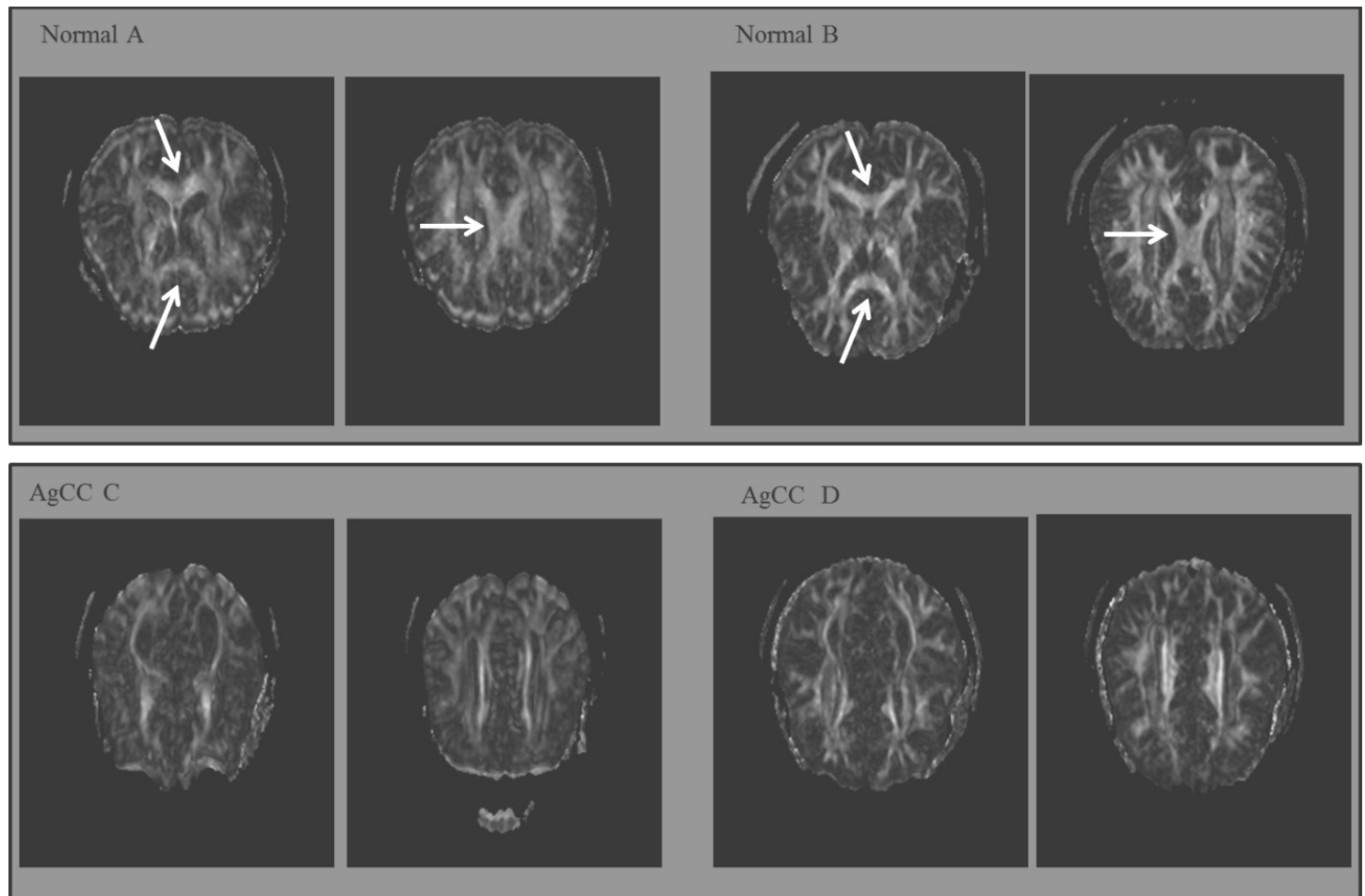

Figure 2. DTI reveals the orientations of white matter tracts: Left-to-right, red; anteroposterior, green; and craniocaudal, purple. DTI of AgCC patients and normal subjects; color FA maps of the white matter tracts are shown. CC was associated with red fiber tracts on axial images. The upper arrow in the CC genu indicates the anterior ROls, the lower arrow in the CC splenium the posterior CC ROIs, and the middle arrow in the body the bodily ROls. The FA threshold was 0.2 and the angle threshold $50^{\circ}$.

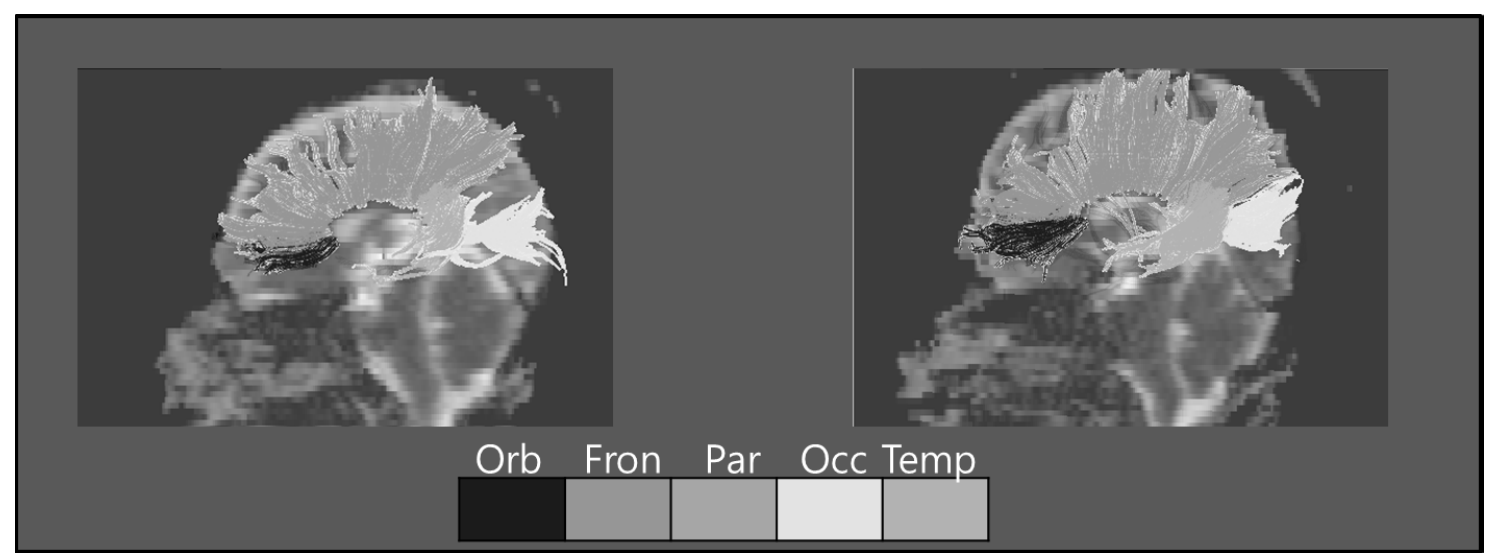

Figure 3. DTI of representative callosal fibers of a normal subject. The fibers project toward the orbital lobe (blue), frontal lobe (green), parietal lobe (orange), occipital lobe (yellow), and temporal lobe (cyan). 
skills. ${ }^{13)}$ The clinical outcomes vary. Interhemispheric connections may become re-established via the AC or other networks. ${ }^{14)}$ Most patients with primary AgCC lack AC involvement. Persistence of the AC alone may not explain the residual interhemispheric influences, but the AC may engage in compensatory adaptive plasticity (recruitment of AC fibers). In our cases, MRI revealed ACs but no CCs. DTI, an extension of diffusion weighted imaging (DWI), takes advantages of the brain's limited perpendicular water motion to fiber tracts but less constrained motion in the direction of fiber tracts. Conventional structural imaging techniques such as computed tomography (CT) and MRI make it possible to demonstrate whether such structurally intact region is functionally normal. DTI can help to identify disconnected regions and determine the extent of white matter damage post stroke. ${ }^{3)}$ DTI provides imaging of axonal pathways in the living brain and provides information about tissue microstructure by measuring fractional anisotropy (FA). ${ }^{4}$ No standard AgCC treatment exists; it is important to explore the impact of AgCC on overall function and prescribe (possibly) rehabilitative interventions. Usually, symptoms and seizures are managed as they occur. Chiappedi and Maurizio suggested that physiotherapy may reduce motor problems and their sequelae in patients with complex neurodevelopmental disorders, as may motor, cognitive, and relational training. ${ }^{13)}$ Two cases did not show any physical problems but having the cognitive dysfunction. Especially, they were able to have a memory registration but not able to recall of memory. Rehabilitative treatments affect brain networking (and even brain shape); training intensity and duration must be appropriate. Plasticity refers to the ability of the brain to change based on new inputs; plasticity may be enhanced by training even in older adults. Early diagnosis and therapy of/for AgCC patients, with a focus on right/left co-ordination, render the condition much more manageable than is otherwise the case. Rapid diagnosis and treatment may improve social and developmental outcomes. Here, we described the MRI and DTI findings of our patients; we did not explore movement in detail. We did not perform coronal MRI, and we did not examine the cingulum or Probst bundles.

\section{Funding information and Acknowledgement}

The authors wish to acknowledge the financial support of the St.Vincent's hospital, research institute of medical science foundation (52019B000100176)

\section{References}

1. Norman M, Mc Gillivray B, Kalousek D. Congenital malformations of the brain. Oxford University Press, New York, 1995.

2. Davila-Gutierrez G. Agenesis and dysgenesis of the corpus callosum. Semin Pediatr Neurol. 2002;9(4): 292-301.

3. Le Bihan D. Looking into the functional architecture of the brain with diffusion mri. Nat Rev Neurosci. 2003;4(6):469-80.

4. Basser PJ, Pierpaoli C. Microstructural and physiological features of tissues elucidated by quantitative-diffusiontensor mri. J Magn Reson B. 1996;111(3):209-19.

5. Kim Y, Kim SH, Kim JS, et al. Modification of cerebellar afferent pathway in the subacute phase of stroke. J Stroke Cerebrovasc Dis. 2018;27(9):2445-52.

6. Mori S, Crain BJ, Chacko VP, et al. Three-dimensional tracking of axonal projections in the brain by magnetic resonance imaging. Ann Neurol. 1999;45(2):265-9.

7. Jang SH, Kim K, Kim SH, et al. The relation between motor function of stroke patients and diffusion tensor imaging findings for the corticospinal tract. Neurosci Lett. 2014;572:1-6.

8. Jiang H, van Zijl PC, Kim J, et al. Dtistudio: Resource program for diffusion tensor computation and fiber bundle tracking. Comput Methods Programs Biomed. 2006;81(2):106-16.

9. Huang H, Zhang J, Jiang H, et al. Dti tractography based parcellation of white matter: Application to the mid-sagittal morphology of corpus callosum. Neuroimage. 2005;26(1):195-205.

10. Tomasch J. Size, distribution, and number of fibres in the human corpus callosum. Anat Rec. 1954;119(1): 119-35.

11. Bloom JS, Hynd GW. The role of the corpus callosum in interhemispheric transfer of information: Excitation or inhibition?. Neuropsychol Rev. 2005;15(2):59-71. 
12. Gazzaniga MS. Cerebral specialization and interhemispheric communication: Does the corpus callosum enable the human condition? Brain. 2000;123 (7):1293-326.

13. Chiappedi M, Bejor M. Corpus callosum agenesis and rehabilitative treatment. Ital J Pediatr. 2010;36:64.
14. Brown WS, Jeeves MA, Dietrich R, et al. Bilateral field advantage and evoked potential interhemispheric transmission in commissurotomy and callosal agenesis. Neuropsychologia. 1999;37(10):1165-80. 
